Supplementary Material

\title{
An Assessment of the Role of Quenched Randomness in the Stereochemical Sequences of Atactic Vinyl Polymers
}

\author{
Wayne L. Mattice and Numan Waheed \\ Institute of Polymer Science \\ The University of Akron \\ Akron, Ohio 44325-3909
}

The supplementary material presents the 32 statistical weight matrices, $\mathbf{U}_{\text {abcde }}$, for the optimized first approximation virtual bond RIS model of polystyrene. Each of the letters in the subscript (abcde) may be either $d$ or $l$. They denote the pseudoasymmetric centers at five consecutive $\mathrm{C}^{\alpha}$. The rows are indexed by conformations of the six main chain $\mathrm{C}-\mathrm{C}$ bonds in the three diads represented by abcd, and the columns are indexed by the conformations of the six main chain C-C bonds in bcde. The conformations are denoted by 1,2 , or 3 , for $t, g^{+}, g^{-}$. For each $\mathbf{U}_{\text {abcde, }}$, the nonzero elements are presented one to a line. The format of a line is row, column, index for the row, index for the column, $l(\mathrm{~nm}), \theta\left(^{\circ}\right), \phi\left(^{\circ}\right)$, statistical weight. If the conformation at the last two diads of TPN with stereochemical sequence abcd is not included also in the conformations allowed for the first two diads of TPN with stereochemical sequence bcde, where both sets of conformations are selected via the requirements for the firstapproximation model, there will be a null row in $\mathbf{U}_{\text {abcde, }}$, thereby preventing the propagation of some of the conformations. Null rows and columns do not appear in the first-approximation treatments of the isotactic and syndiotactic chains, but they do appear in a few statistical weight matrices required for the atactic chains. The occurrence of null rows and columns is noted at appropriate entries in the list that follows.

$\mathbf{U}_{d d d d d}, 14$ rows, 14 columns. Reversal of signs for all $\phi$, and exchange of 2 and 3 in the indices for the rows and columns, yields $\mathbf{U}_{l l l l}$. 
$\mathbf{U}_{d d d d l}, 14$ rows, 4 columns, 7 null rows. Reversal of signs for all $\phi$, and exchange of 2 and 3 in the indices for the rows and columns, yields $\mathbf{U}_{\text {lllld. }}$

\footnotetext{
$1,1,11-13-13,13-13-11,0.64,42,103, \eta$ $7,1,13-13-13,13-13-11,0.64,42,103, \eta$ $11,1,21-13-13,13-13-11,0.64,42,103, \eta$ $1,2,11-13-13,13-13-12,0.64,70,56, \eta$ 7, 2, 13-13-13, 13-13-12, 0.64, 70, 56, ๆ $11,2,21-13-13,13-13-12,0.64,70,56, \eta$ 2, 3, 11-21-13, 21-13-11, 0.64, 42, 144, $\eta$ 13, 3, 21-21-13, 21-13-11, 0.64, 42, 144, $\eta$ $3,4,11-21-21,21-21-11,0.64,91,-50, \eta$ $14,4,21-21-21,21-21-11,0.64,91,-50, \eta$
} 
$\mathbf{U}_{\text {dddld }}, 4$ rows, 5 columns, one null row and column. Reversal of signs for all $\phi$, and exchange of 2 and

3 in the indices for the rows and columns, yields $\mathbf{U}_{\text {lld }}$

$1,1,13-13-11,13-11-11,0.54,55,-154, \eta^{2}$
$3,1,21-13-11,13-11-11,0.54,55,-154, \eta^{2}$
$1,2,13-13-11,13-11-33,0.54,78,-107, \eta^{2}$
$3,2,21-13-11,13-11-33,0.54,78,-107, \eta^{2}$
$4,3,21-21-11,21-11-11,0.54,55,-146, \eta^{2}$
$4,4,21-21-11,21-11-33,0.54,78,-104, \eta^{2}$

$\mathbf{U}_{d d d l l}, 4$ rows, 5 columns. One null row and column. Reversal of signs for all $\phi$, and exchange of 2 and 3 in the indices for the rows and columns, yields $\mathbf{U}_{\text {llldd }}$

$1,1,13-13-11,13-11-12,0.54,91,-122, \eta^{2}$
$3,1,21-13-11,13-11-12,0.54,91,-122, \eta^{2}$
$1,2,13-13-11,13-11-31,0.54,42,-129, \eta^{2}$
$3,2,21-13-11,13-11-31,0.54,42,-129, \eta^{2}$
$4,3,21-21-11,21-11-12,0.54,91,-118, \eta^{2}$
$4,4,21-21-11,21-11-31,0.54,42,-122, \eta^{2}$

$\mathbf{U}_{\text {ddldd }}, 5$ rows, 5 columns. Reversal of signs for all $\phi$, and exchange of 2 and 3 in the indices for the rows and columns, yields $\mathbf{U}_{\text {lldll }}$

$1,1,13-11-11,11-11-13,0.54,91,146, \eta^{2}$
$3,1,21-11-11,11-11-13,0.54,91,146, \eta^{2}$
$1,2,13-11-11,11-11-21,0.54,42,154, \eta^{2}$
$3,2,21-11-11,11-11-21,0.54,42,154, \eta^{2}$
$2,3,13-11-33,11-33-13,0.73,80,-21,1$
$4,3,21-11-33,11-33-13,0.73,80,-21,1$
$5,4,21-22-11,22-11-13,0.54,91,104, \eta^{2}$
$5,5,21-22-11,22-11-21,0.54,42,107, \eta^{2}$

$\mathbf{U}_{\text {ddldl, }} 5$ rows, 4 columns. Reversal of signs for all $\phi$, and exchange of 2 and 3 in the indices for the rows and columns, yields $\mathbf{U}_{\text {lldld }}$ 
$1,1,13-11-11,11-11-11,0.54,55,180, \eta^{2}$
$3,1,21-11-11,11-11-11,0.54,55,180, \eta^{2}$
$1,2,13-11-11,11-11-22,0.54,78,136.5, \eta^{2}$
$3,2,21-11-11,11-11-22,0.54,78,136.5, \eta^{2}$
$2,3,13-11-33,11-33-11,0.73,78,-22.5,1$
$4,3,21-11-33,11-33-11,0.73,78,-22.5,1$
$5,5,21-22-11,22-11-11,0.54,55,136.5, \eta^{2}$

$\mathbf{U}_{\text {ddlld, }} 5$ rows, 4 columns. Reversal of signs for all $\phi$, and exchange of 2 and 3 in the indices for the rows and columns, yields $\mathbf{U}_{l l d d}$

$1,1,13-11-12,11-12-11,0.64,42,-97, \eta$
$3,1,21-11-12,11-12-11,0.64,42,-97, \eta$
$2,2,13-11-31,11-31-11,0.64,91,97, \eta$
$4,2,21-11-31,11-31-11,0.64,91,97, \eta$
$2,3,13-11-31,11-31-33,0.64,80,144, \eta$
$4,3,21-11-31,11-31-33,0.64,80,144, \eta$
$5,4,21-22-12,22-12-11,0.64,42,-144, \eta$

$\mathbf{U}_{\text {ddlll }}, 5$ rows, 4 columns. One null row and column. Reversal of signs for all $\phi$, and exchange of 2 and 3 in the indices for the rows and columns, yields $\mathbf{U}_{\text {llddd }}$

$1,1,13-11-12,11-12-12,0.64,70,-50, \eta$

$3,1,21-11-12,11-12-12,0.64,70,-50, \eta$

$2,2,13-11-31,11-31-12,0.64,100,144, \eta$

$4,2,21-11-31,11-31-12,0.64,100,144, \eta$

$2,3,13-11-31,11-31-31,0.64,70,103, \eta$

$4,3,21-11-31,11-31-31,0.64,70,103, \eta$

$\mathbf{U}_{\text {dlddd }}, 5$ rows, 4 columns. One null row and column. Reversal of signs for all $\phi$, and exchange of 2 and 3 in the indices for the rows and columns, yields $\mathbf{U}_{\text {ldlll }}$

$1,1,11-11-13,11-13-13,0.64,70,50, \eta$

$4,1,22-11-13,11-13-13,0.64,70,50, \eta$

$2,2,11-11-21,11-21-13,0.64,100,-144, \eta$

$5,2,22-11-21,11-21-13,0.64,100,-144, \eta$

$2,3,11-11-21,11-21-21,0.64,70,-103, \eta$ 
$5,3,22-11-21,11-21-21,0.64,70,-103, \eta$

$\mathbf{U}_{\text {dlddl, }}, 5$ rows, 4 columns. Reversal of signs for all $\phi$, and exchange of 2 and 3 in the indices for the rows and columns, yields $\mathbf{U}_{l d l d}$

$1,1,11-11-13,11-13-11,0.64,42,97, \eta$

$4,1,22-11-13,11-13-11,0.64,42,97, \eta$

$2,2,11-11-21,11-21-11,0.64,91,-97, \eta$

$5,2,22-11-21,11-21-11,0.64,91,-97, \eta$

$2,3,11-11-21,11-21-22,0.64,80,-144, \eta$

$5,3,22-11-21,11-21-22,0.64,80,-144, \eta$

$3,4,11-33-13,33-13-11,0.64,42,144, \eta$

$\mathbf{U}_{\text {dldld, }} 4$ rows, 4 columns. Reversal of signs for all $\phi$, and exchange of 2 and 3 in the indices for the rows and columns, yields $\mathbf{U}_{l d l d l}$

$1,1,11-11-11,11-11-11,0.54,55,180, \eta^{2}$
$4,1,22-11-11,11-11-11,0.54,55,180, \eta^{2}$
$1,2,11-11-11,11-11-33,0.54,78,-136.5, \eta^{2}$
$4,2,22-11-11,11-11-33,0.54,78,-136.5, \eta^{2}$
$2,3,11-11-22,11-22-11,0.73,78,-22.5,1$
$3,4,11-33-11,33-11-11,0.54,55,-136.5, \eta^{2}$

$\mathbf{U}_{\text {dldll, }} 4$ rows, 5 columns. Reversal of signs for all $\phi$, and exchange of 2 and 3 in the indices for the rows and columns, yields $\mathbf{U}_{l d l d}$

1, 1, 11-11-11, 11-11-12, 0.54, 91,-146, $\eta^{2}$
4, 1, 22-11-11, 11-11-12, 0.54, 91,-146, $\eta^{2}$
1, 2, 11-11-11, 11-11-31, 0.54, 42,-154, $\eta^{2}$
4, 2, 22-11-11, 11-11-31, 0.54, 42,-154, $\eta^{2}$
2, 3, 11-11-22, 11-22-12, 0.73, 80, 21,
3, 4, 11-33-11, 33-11-12, 0.54, 91,-104, $\eta^{2}$
$3,5,11-33-11,33-11-31,0.54,42,-107, \eta^{2}$ 
$\mathbf{U}_{\text {dlldd }}, 4$ rows, 5 columns. Reversal of signs for all $\phi$, and exchange of 2 and 3 in the indices for the rows and columns, yields $\mathbf{U}_{\text {lddll }}$

$$
\begin{aligned}
& 1,1,11-12-11,12-11-13,0.54,91,122, \eta^{2} \\
& 4,1,22-12-11,12-11-13,0.54,91,122, \eta^{2} \\
& 1,2,11-12-11,12-11-21,0.54,42,129, \eta^{2} \\
& 4,2,22-12-11,12-11-21,0.54,42,129, \eta^{2} \\
& 2,3,11-31-11,31-11-13,0.54,91,118, \eta^{2} \\
& 2,4,11-31-11,31-11-21,0.54,42,122, \eta^{2} \\
& 3,5,11-31-33,31-33-13,0.73,80,-73,1
\end{aligned}
$$

$\mathbf{U}_{\text {dlldl }}, 4$ rows, 5 columns. Reversal of signs for all $\phi$, and exchange of 2 and 3 in the indices for the rows and columns, yields $\mathbf{U}_{\text {lddld }}$

$$
\begin{aligned}
& 1,1,11-12-11,12-11-11,0.54,55,154, \eta^{2} \\
& 4,1,22-12-11,12-11-11,0.54,55,154, \eta^{2} \\
& 1,2,11-12-11,12-11-22,0.54,78,107, \eta^{2} \\
& 4,2,22-12-11,12-11-22,0.54,78,107, \eta^{2} \\
& 2,3,11-31-11,31-11-11,0.54,55,146, \eta^{2} \\
& 2,4,11-31-11,31-11-22,0.54,78,104, \eta^{2} \\
& 3,5,11-31-33,31-33-11,0.73,78,-21,1
\end{aligned}
$$

$\mathbf{U}_{\text {dllld }}, 4$ rows, 4 columns. Reversal of signs for all $\phi$, and exchange of 2 and 3 in the indices for the rows and columns, yields $\mathbf{U}_{l d d d l}$

$$
\begin{aligned}
& 1,1,11-12-12,12-12-11,0.64,42,-103, \eta \\
& 1,2,11-12-12,12-12-13,0.64,70,-56, \eta \\
& 2,3,11-31-12,31-12-11,0.64,42,-144, \eta \\
& 3,4,11-31-31,31-31-11,0.64,91,50, \eta \\
& 4,4,21-31-31,31-31-11,0.64,91,50, \eta
\end{aligned}
$$

$\mathbf{U}_{\text {dlll }}, 4$ rows, 14 columns, 7 null columns. Reversal of signs for all $\phi$, and exchange of 2 and 3 in the indices for the rows and columns, yields $\mathbf{U}_{\text {ldddd }}$

$$
1,6,11-12-12,12-12-11,0.64,84,-83, \eta
$$


$1,7,11-12-12,12-12-12,0.64,70,-58, \eta$

$2,10,11-31-12,31-12-11,0.64,84,-123, \eta$

$2,11,11-31-12,31-12-12,0.64,70,-98, \eta$

$3,12,11-31-31,31-31-11,0.64,145,98, \eta$

4, 12, 21-31-31, 31-31-11, 0.64, 145, 98, $\eta$

$3,13,11-31-31,31-31-12,0.64,100,98, \eta$

$4,13,21-31-31,31-31-12,0.64,100,98, \eta$

$3,14,11-31-31,31-31-31,0.64,70,58, \eta$

$4,14,21-31-31,31-31-31,0.64,70,58, \eta$ 